\title{
Exploring human factors issues for urban air mobility operations
}

\author{
Tamsyn Edwards ${ }^{1}$ \\ San Jose State University at NASA Ames Research Center, Moffett Field, CA, 94035 \\ and \\ Savita Verma ${ }^{2}$ \\ NASA Ames Research Center, Moffett Field, CA, 94035 \\ and \\ Jillian Keeler ${ }^{3}$ \\ NASA Ames Research Center, Moffett Field, CA, 94035
}

\begin{abstract}
Urban air mobility (UAM) is currently receiving increased attention in the aviation literature as a new entrant into the airspace. Although the introduction of UAM offers the potential for significant benefits, it also creates the potential for fundamental change to the current air traffic management system. Several concepts are being explored to enable the development of a safe and efficient UAM system for near, mid and far term operations. A concept of operations for near term operations proposes several assumptions. Concepts for roles and responsibilities of human operators such as air traffic controllers propose different degrees of involvement. Identifying and exploring human factors issues is therefore a critical next step in the forward progression of concept development. A human in the loop air traffic control simulation was used to investigate the effect of UAM traffic density and changes in current airspace routes and communication procedures on subjective controller workload and efficiency-related task performance. Findings indicate that although subjective workload was manageable for low density operations, medium and high density operations led to unmanageable levels of workload, leading to refusals to allow more vehicles into controlled airspace. By implementing a letter of agreement, verbal communications were reduced which were associated with reduced workload. Optimized routes were also associated with reduced workload and increased performance efficiency. Although these adjustments can positively support controller performance, workload still remained high during the high density UAM traffic scenarios. It is therefore suggested that, in order for UAM operation to become scalable, human operators will be required to work differently compared to current air traffic controllers. Future research should focus on the level and type of human operator or controller involvement and interaction with automated systems, to ensure safety and efficiency within UAM operations.
\end{abstract}

\footnotetext{
${ }^{1}$ Senior Research Associate, Human Systems Integration Division, NASA Ames Research Center, Mail Stop 262-4, Moffett Field, CA 94043, AIAA Senior Member.

${ }^{2}$ Senior Researcher, Aerospace High Density Operations Branch, NASA Ames Research Center, Mail Stop 210-4, Moffett Field, CA 94043.

${ }^{3}$ Researcher, Human Systems Integration Division, NASA Ames Research Center, Mail Stop 262-4, Moffett Field, CA 94043.
} 


\section{Introduction}

$\mathrm{O}$ $\mathrm{NE}$ of the challenges facing the current air traffic management system is the series of new entrants that are seeking integration into the airspace. As a potential new entrant, urban air mobility (UAM), is currently receiving increased attention in the aviation literature as a service-oriented option to avoid congestion in metropolitan areas $[1,2]$. UAM has been defined by the NASA ATM-X project as "a safe and efficient system for air passenger and cargo transportation within an urban area" [3, p.3366). Technological advancements, in combination with falling costs and ride-share business models [2], have facilitated the exploration of UAM as a feasible solution to transporting people and goods around metropolitan areas at greater speed and efficiency [4]. Initial concepts include small package delivery and other urban UAS services, as well as passenger-carrying vehicles [3]. It is envisaged that passenger transport would be focused on high-density metropolitan areas, and rely on fleets of small vehicles carrying 2-6 passengers, focusing on short-distance flights $[2,3]$.

Although the introduction of UAM offers the potential for significant benefits, such as increased efficiency for customers [4], it also creates the potential for fundamental change to the current air traffic management system. It has been acknowledged in the literature that in addition to technical challenges, including those associated with UAM vehicles such as ride quality and energy efficiency, barriers to integration of UAM operations in the existing airspace must be considered and mitigated [e.g. 3] to enable a safe and efficient integration with the current system. Documented challenges include modifications to the airspace [4] airspace allocation, demand on human operators, and interactions with traditional airspace users (such as general aviation and commercial aircraft) [5].

In order to facilitate concept development for the safe and efficient integration of UAM vehicles and operations into the National Air Space, the National Aeronautics and Space Administration (NASA) will "develop detailed concepts of operations for UAM airspace integration at different stages of operational maturity" [1, p3678]. Phase 1 proposes development of a concept of operations for near term operations. Several assumptions are made in this near-term stage. UAM vehicles will be low-density, and will be subject a small set of fixed routes that primarily focus on the current-day helicopter routes around metropolitan areas [1]. In addition, at least for the near term, UAM vehicles are envisaged to be subject to the existing regulations of air traffic. One of the implications of this is that UAM vehicles will be expected to abide by the regulations around clearances into controlled airspace [1]. In current controlled airspace, (Class A- D), each class has a set of regulations for the aircraft using the airspace. As the scope of UAM operations focus primarily on metropolitan areas, UAM vehicles are likely to be operating in controlled airspace. For near-term operations therefore, UAM vehicles must adhere to the regulations as required for any other air traffic. Specifically, these regulations state that UAM flights would be required to communicate with ATC prior to entering Class B, C, or D airspace [3] as well as gain a flight clearance prior to take-off within controlled airspace. Regarding the roles and responsibilities of human operators such as air traffic controllers, there are currently various concepts from several organization that propose different degrees of involvement. However, the current regulations for controlled airspace has obvious implications for task demand for current human operators in the system, as well as efficiency for UAM flights. Identifying and exploring such human factors issues is therefore a critical next step in the forward progression of this project. In addition, exploring potential human factors issues during this early phase results in the opportunity to identify and mitigate potential limiting factors for UAMs (such as efficiencies, capacity) as well as potential risks to the human operator (such as overload) or safety issues (such as separation or interactions between UAM flights and other airspace users.

The current research aimed to contribute further understanding to the human factors considerations for near-term UAM concept development, with the potential to influence future design of UAM concept of operations to maximize safety and efficiency. The current research is conducted as part of the NASA ATM-X project, and as such, is conducted in line with the ATM-X project goals and continues to apply the Phase 1 assumptions outlined by $[1,3]$. The research reported in this paper has three specific aims. First, the research aimed to investigate different levels of UAM-related traffic demand on air traffic controllers in class b and $c$ airspace. A second aim was to investigate initial tools and information that controllers may need. The final aim was to explore different route and communication agreements in order to determine changes on demand. To address these aims, a human in the loop simulation was conducted with operational Tower-based controllers from the Dallas areas, including Dallas Fort 
Worth, Dallas Love Field and Addison Towers, utilizing the Dallas metropolitan downtown airspace for simulation scenarios.

\section{Method}

\section{A. Design overview}

A human in the loop simulation of air traffic control tower positions was conducted to investigate the effect of UAM traffic demand, optimized routes and communication procedures on self-reported controller workload and efficiency-related performance. The simulation was centered on low-altitude tower control sectors in the North Texas Metroplex area. The study used a mixed measures design. Control position served as the between-measures independent variable and consisted of three levels; Dallas ft Worth (DFW) Local East 3 position (south flow), Dallas Love Field (DAL) helicopter ('helo') position, and Addison tower (ADS) local position. Three within subjects variables were utilized. Task demand was manipulated to create three simulation scenarios, consisting of low, medium and high density UAM traffic. Two forms of communication procedure were utilized as the second variable, specifically, current day communication procedures or reduced verbal communications procedure implemented via a letter- of-agreement (LOA). Finally, the routes available to UAM traffic were manipulated, and consisted of two levels - the use of current day helicopter routes, or modified routes that were optimized for UAM vehicles. The study did not use a full-factorial design. A total of 9 conditions were completed by each control position. Participants were six recently-retired controllers who had previously worked in tower control. Two controllers participated in each control position. Self-reported workload was measured throughout each simulation at 4-minute intervals using a 1-5 rating scale which appeared at the top of the radar screen. Efficiency-related performance was inferred from the number of UAM vehicles controlled in each simulation and percentage of total UAM vehicles that were accepted into controlled airspace. Pseudo pilots were paired with controllers and completed standard pilot tasks such as controlling the aircraft in accordance with controller instructions and communicating with controllers. Each simulation session lasted for 40 minutes.

\section{B. Airspace}

Participants were asked to control airspace in the North Dallas, TX metroplex area, surrounding three airports located in Dallas, Texas, which was observed to be a particularly complex sector given the mix of traffic transiting airspace and airspace sectorization between three control towers. Specially, participants controlled low altitude sectors from the East tower position at Dallas Fort Worth International Airport (DFW) Dallas Love Field tower airspace, and Addison tower airspace.

\section{Experimental conditions}

\section{Between-measures variable: Controller position}

This study utilized one between-measures variable, and three within-measures variables in order to investigate the effect of UAM traffic demand, optimized routes and communication procedures on self-reported controller workload and efficiency-related performance. The between measures variable was defined by three independent air traffic control positions, DFW Local East 3 position (south flow), DAL helicopter ('helo') position, and ADS local position. DFW Local East 3 was responsible for UAM vehicles departing and arriving on 17L arrivals, as well as UAM traffic on routes that are in his sector boundaries. (see section). Dallas Love Field (DAL) helicopter ('helo') position was responsible for only for all UAM traffic in the sectors of control, and Addison tower (ADS) local position was responsible for UAM traffic in addition to VFR traffic and IFR traffic. All control positions were required to complete a set of tasks in relation to controlling UAM traffic which are described in detail in section XX procedure. Two participants were assigned work to each controller position.

\section{Within-measures variable: UAM traffic density}

UAM traffic density was manipulated in order to change taskload. Density was manipulated by increasing UAM traffic count, and reducing the spacing distance and time between each UAM aircraft. Three levels of traffic density created, generating three different experimental scenarios, defined in Table XX 


\begin{tabular}{lccc}
\hline \multicolumn{1}{c}{ Scenario } & $\begin{array}{c}\text { Temporal } \\
\text { spacing } \\
\text { (seconds) }\end{array}$ & $\begin{array}{c}\text { Distance } \\
\text { spacing (miles) }\end{array}$ & UAM Count \\
\hline $\begin{array}{l}\text { Scenario 1: Low UAM } \\
\text { density }\end{array}$ & 90 & 3.75 & 115 \\
$\begin{array}{l}\text { Scenario 2: Medium UAM } \\
\text { density }\end{array}$ & 60 & 2.5 & 167 \\
$\begin{array}{l}\text { Scenario 3: High UAM } \\
\text { density }\end{array}$ & 45 & 1.88 & 225 \\
\hline
\end{tabular}

Background traffic, specifically, simulations of aircraft using visual flight rules (VFR) and commercial aircraft using instrument flight rules, were included in each scenario based on current day traffic levels. Bac ground traffic numbers remained constant across scenarios for each controller positions.

\section{Within measures variable - Communication procedures}

Two sets of communication procedures were used in this study. The first replicated current day communication procedures for entering/exiting controlled airspace and taking off and landing at airports within controlled airspace, and was labelled 'current day communications'. The condition assumed no letter of agreement, or reduced communication requirements, between UAM companies and control facilities and no ATIS broadcast with UAM traffic information. Controllers were required to perform tasks, which are representative of current day tasks for VFR traffic. Specifically, controllers were asked to: assign beacon codes, assign altitude and speed, make traffic calls to both commercial and UAM traffic where necessary, issue advisories for takeoff and clearance to enter Class B airspace (e.g. "UAM942, Love Tower, cleared to enter class bravo. Squawk 4043 [additional instructions]") hand-off traffic to other sectors and receive handoffs. UAM pilots called for clearance to take off or enter Class B airspace (e.g. "UAM123, cleared to enter class bravo, landing at vertiport will be at your own risk"). Controllers were able to approve requests and issue a clearance, or could choose to refuse entry by stating "unable". The second set of communication procedures simulated a letter of agreement (LOA) between UAM companies and control facilities. LOAs help reduce verbiage and therefore time spent on verbal communications. The LOA was used to create standardized routes which UAMs used depending on departure point and route. Each route was preassigned information used as beacon codes, altitudes and speeds, so that controllers did not need to pas this information verbally, unlike the no LOA communication procedure. ATIS was used to broadcast UAM traffic locations. Controllers gave clearances using route names, in which clearance to class $\mathrm{b}$ airspace, speed, altitude and beacon code were implicit. For example, when entering Class B airspace, communication procedure was as follows: UAM Entering Class B airspace "UAM173, Love Tower, cleared via [route name]". For departure, the standard communication phraseology became "UAM123 [Airport] Tower, cleared via [route name]" (departure is implicit) Again, Controllers were able to approve requests and issue a clearance, or could choose to refuse entry by stating "unable". A full comparison of the differences between COMMUNCIATION SETS IS presented in table xx

\begin{tabular}{|l|l|l|}
\hline & Current Routes Without LOA & Current Routes with LOA \\
\hline Routes & $\begin{array}{l}\text { Current Helo routes, controller assigns } \\
\text { altitudes }\end{array}$ & $\begin{array}{l}\text { Current Helo routes with transition waypoints and } \\
\text { altitudes defined in LOA }\end{array}$ \\
\hline $\begin{array}{l}\text { Beacon } \\
\text { code } \\
\text { assignment }\end{array}$ & Verbally communicated by controller & Pre assigned via LOA \\
\hline $\begin{array}{l}\text { Route } \\
\text { Clearance }\end{array}$ & $\begin{array}{l}\text { Pilot requests full route clearance by } \\
\text { describing the intended route }\end{array}$ & $\begin{array}{l}\text { Pilots request route using route name defined in } \\
\text { LOA }\end{array}$ \\
\hline $\begin{array}{l}\text { Class B } \\
\text { Airspace } \\
\text { Clearance }\end{array}$ & Explicit clearance is required & Implicit in route clearance \\
\hline
\end{tabular}




\begin{tabular}{|l|l|l|}
\hline $\begin{array}{l}\text { Handoffs } \\
(\mathrm{HO})\end{array}$ & $\begin{array}{l}\text { Manual Handoff for flights going out of } \\
\text { sector with usual communication } \\
\text { Communication: “leaving CBA, squawk } \\
\text { VFR" }\end{array}$ & $\begin{array}{l}\text { No communication for exiting Class B airspace } \\
\text { (CBA) } \\
\text { Communication required for sectors Handoff }\end{array}$ \\
\hline $\begin{array}{l}\text { Frequency } \\
\text { change }\end{array}$ & $\begin{array}{l}\text { Freq change required to exit Class B } \\
\text { airspace and between sectors }\end{array}$ & $\begin{array}{l}\text { Automatic frequency change when exiting CBA, } \\
\text { but approval required for sector frequency } \\
\text { change within CBA }\end{array}$ \\
\hline Point Outs & Point outs are required where necessary & $\begin{array}{l}\text { Point outs not required for DFW since they were } \\
\text { spelled out in the LOA }\end{array}$ \\
\hline Traffic Calls & $\begin{array}{l}\text { Controllers responsible for separation in } \\
\text { Class B airspace, will make traffic calls as } \\
\text { necessary }\end{array}$ & $\begin{array}{l}\text { Controllers responsible for separation in Class B } \\
\text { airspace, will make traffic calls as necessary } \\
\text { ATIS broadcasts UAM traffic on spine road will } \\
\text { alleviate traffic calls under normal conditions }\end{array}$ \\
\hline
\end{tabular}

\section{Within measures variable - UAM routes}

The final variable was the routes available to the UAM traffic. Two sets of routes were used. The first set of routes were current day routes that helicopters used (figure $\mathrm{xx}$ ). The second set of routes were a modified version of the current helicopter routes. These modified routes (Figure 2) were designed to avoid approach and departure paths for traditional flights, any common Temporary Flight Restrictions (TFRs), heavily populated areas and were shortened to take account of the limited battery power of electric vertical takeoff and landing (eVTOL) aircraft. The modified routes also attempted to create new routes that could be used two-way routes such as (Central and I-30). Appendix 1 lists the routes used during this study, and compares between the two sets of routes.
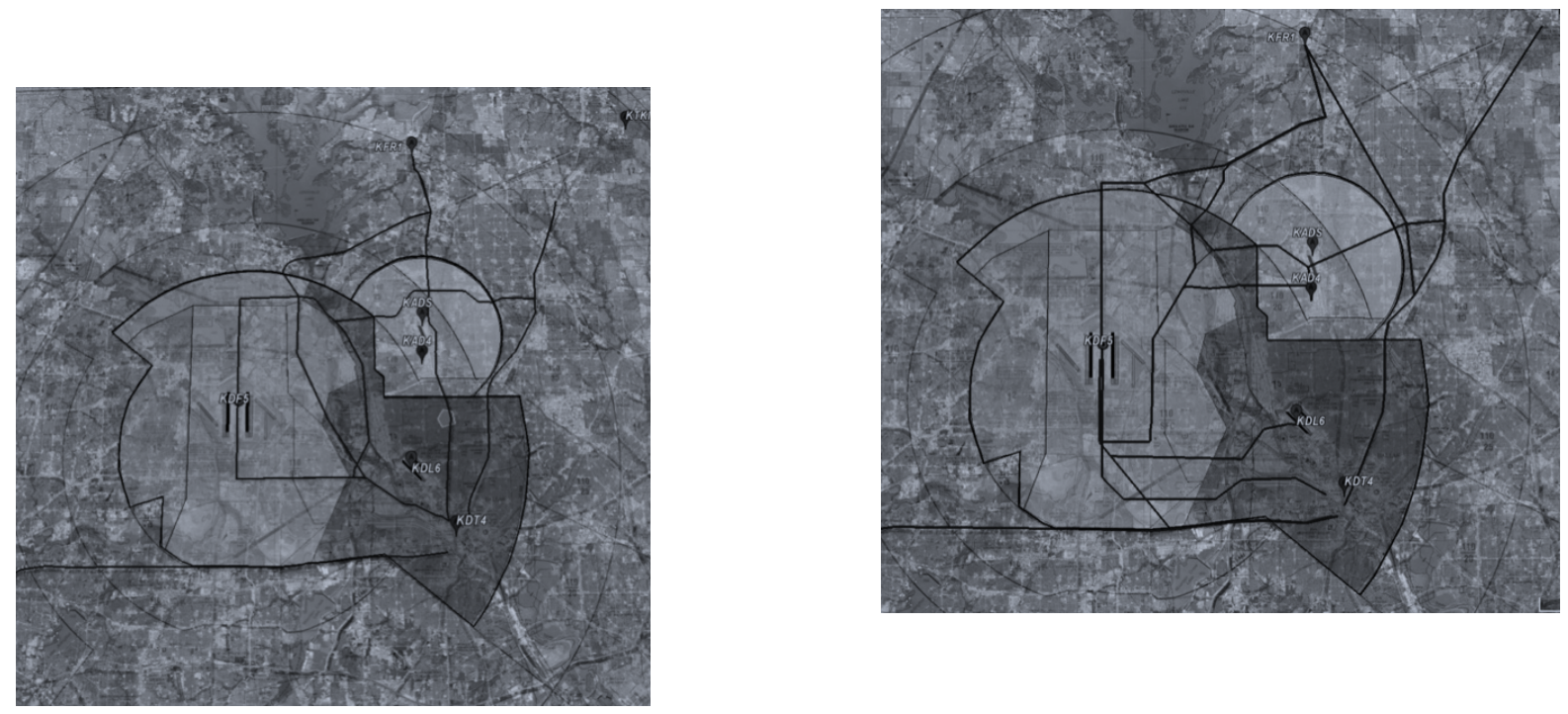

\section{Experimental conditions - Summary}

The three within-measures variables were combined in a non-factorial design to create a total of nine conditions, for each controller position. A baseline condition, with no UAM traffic, was also used, but will not be reported here due to the lack of relevant UAM results.

Conditions 1-3 used Current routes and no LOA (referred to as condition ' $\mathrm{C}$ '). This condition was tested under three different UAM density scenarios. $\mathrm{C} 1$ refers to the condition of current day routes and communication procedures, with no LOA, with the first scenario of low density UAM traffic. C2 used the same routes and procedures, but with medium density UAM traffic. Finally, the same routes and procedures were investigated under high density UAM traffic (C3). 
Conditions 4-6 used Current routes, but this time with a LOA (referred to as conditions 'CL'). Again, the condition was repeated in association with the same 3 scenarios created by different densities of AUM traffic. These conditions are referred to as CL1, CL2, CL3.

Finally, conditions 7-9 used Modified touted (referred to as condition 'M') also with a LOA. The condition was also repeated with the same three traffic densitiy scenarios. Conditions will be referred to throughout this paper as M1, M2, M3.

\section{E. Measures}

The study reported in this paper is part of a larger study [REF]. Only the measures that are relevant to this paper are presented. In line with Edwards' (2012) previous study, the covariate factor of workload was measured using subjective, self-report scales. Mental workload was measured using the uni-dimensional Instantaneous SelfAssessment scale (ISA) (Tattersall \& Foord, 1996). Every 4 minutes, participants were presented with the ISA rating scale at the top of the radar scope and asked to click on the workload rating. Several performance measures were collected during the simulation. For clarity, only one of these performance variables will be examined in this paper: UAM throughput. This variable was selected due to the important efficiency implications and inference of workload of this performance measure. In addition, in contrast to measures such as safety related performance measure number of conflicts accurately detected, this measure allows for greater granularity in performance measurement and can more easily infer performance in simulation settings compared to safety related measures (see Edwards). Measures were recorded continuously in the simulation software.

\section{F. Simulation environment and apparatus}

The simulation was conducted at NASA Ames Research Center's ATC laboratory located in building N210. The software used to emulate the air traffic control system was the Multi-Aircraft Control System (MACS) [7]. Specifically, MACs was used to emulate the Standard Terminal Automation Replacement System (STARS) radar, which is used by TRACON controllers. Participant workstations were configured with a BARCO large-format display and specialized keyboard/ trackball combination that is representative of what is currently used in air traffic control facilities. Voice communications via radio were enabled by a custom, stand-alone system that is also representative of what is used in operations. Data were collected continuously through MACS's data collection processes. For this study, the laboratory was configured to represent DFW Tower, DAL tower and ADS tower via MACS displays. All the traffic outside the towers were handled by "ghost" confederate positions. The pseudo pilots who flew these flights also used MACS in another part of the laboratory. Each simulation session lasted for 40 minutes. UAM traffic were configured to represent a single engine, electric rotorcraft, with Performance profile similar to a Cessna 172 Skyhawk. The speed range was 70-156 knots (indicated airspeed) with a cruise speed of 130 knots. The Speed Range was 70 - 156 knots (indicated airspeed) with a Cruise Speed of 130 knots

\section{G. Participants}

A total of six controllers took part in the simulation, consisting of 4 males and 2 females. Controllers working the DFW and DAL positions were recently retired from DFW tower control. Participant positions for this research effort included DFW Tower Local East-3 or DFW-LE3, DAL Helicopter or DAL Helo position, and ADS Tower controller. Sectors surrounding the positions - DFW Local East-1, DFW Local West, DAL Tower position, and D10 TRACON position were also simulated as confederate positions, although no data were recorded. Demographic information was not recorded.

\section{H. Procedure}

Participants were asked to work the traffic according to the conditions and procedures described in section XX. It was emphasized that the participants could work any of the traffic at any time, as they normally would. controllers were encouraged to not let UAM aircraft enter a sector if they felt it was unsafe to do so or could result in an overload. In addition to the primary tasks, participants were prompted to rate their workload every four minutes for the duration of each run. The study was run over five consecutive days. Half of the first day was devoted to classroom training the participants on the study environment and procedures, with a subsequent half day training on simulated positions prior to each set of conditions (conditions C1-3, conditions CL1-3 and conditions M1-3). After the training, experimental runs were started and data were collected. Beginning in the afternoon of the second day, participants worked 22 data collection runs (21 planned runs and one repeat). Participants completed questionnaires at the end of each run, as well as a post-simulation questionnaire. The last session on the fifth day was a debrief that 
provided an additional opportunity for participants to offer feedback. Data from workstation logs and controller responses were analyzed.

\section{Results}

The results section below compares data across different classification of airspace (characterized by the three control tower positions), the form of communication (current day or LOA) and the type of route that was controlled (current day or modified) to investigate the demands of workload on controllers under each condition and the association with a measure of efficiency performance. In order to understand the impact on integrating UAM into controlled airspace in a near term environment.

\section{A. Subjective workload}

Subjective workload was measured throughout the study at 4 minute intervals, resulting in 10 workload scores across the 40 minute run. Average workload ratings for each condition, as well as for individual and collapsed controller positions, are presented in Table 1, and will be referred to throughout sections $\mathrm{xx}-\mathrm{xx}$

Table 1. Mean and standard deviation for workload (as rated by ISA) for all conditions and controller positions.

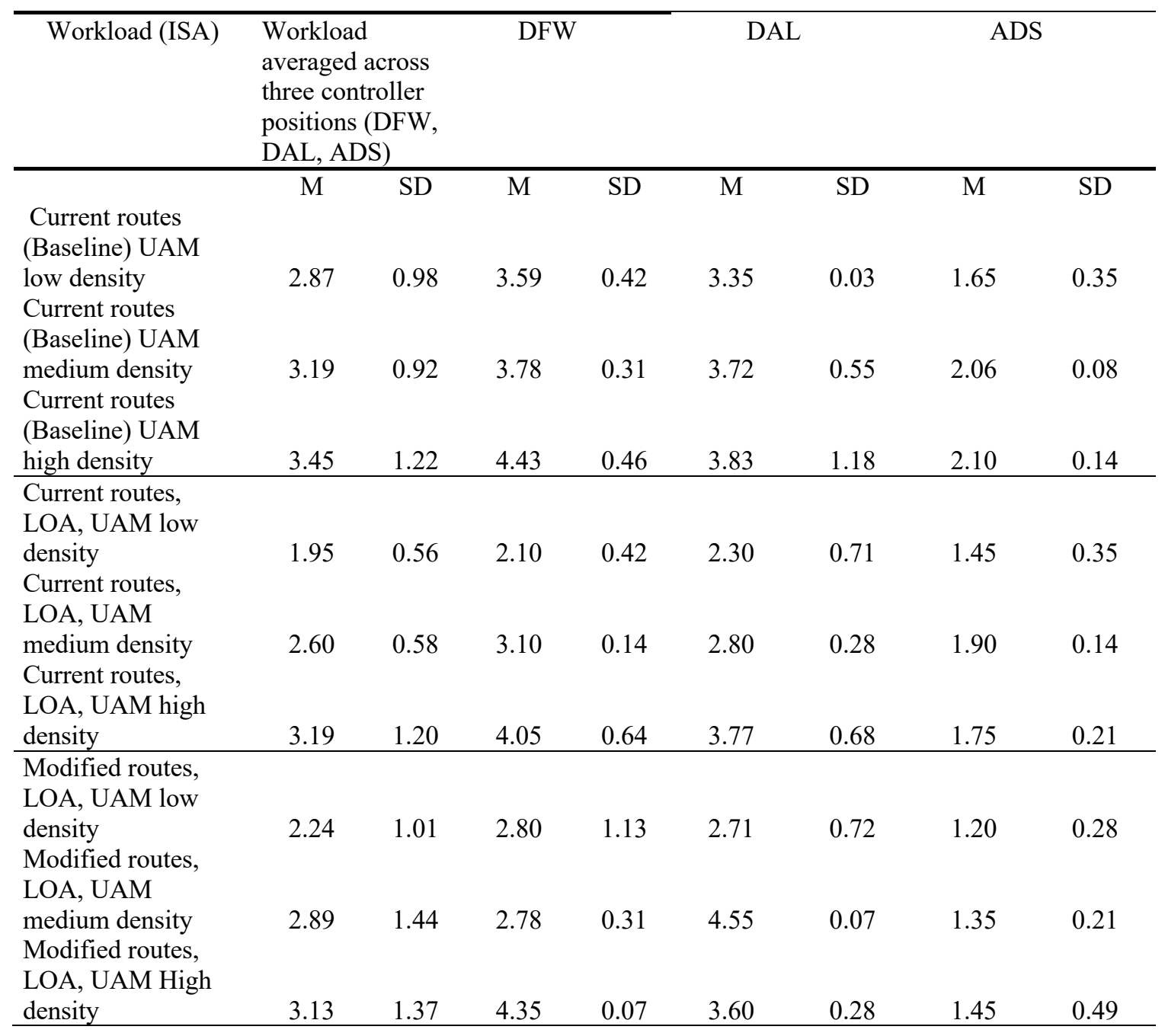


1. Main effect of density: Subjective workload across UAM density conditions (taskload and workload)

Subjective ratings of workload were considered in relation to UAM vehicle density. In order to gain an overview of the workload data trends, workload ratings were first averaged across the three controller positions included in the study: Dallas ft Worth Local East 3 position, Dallas Love Field Helo position, and Addison Tower position.

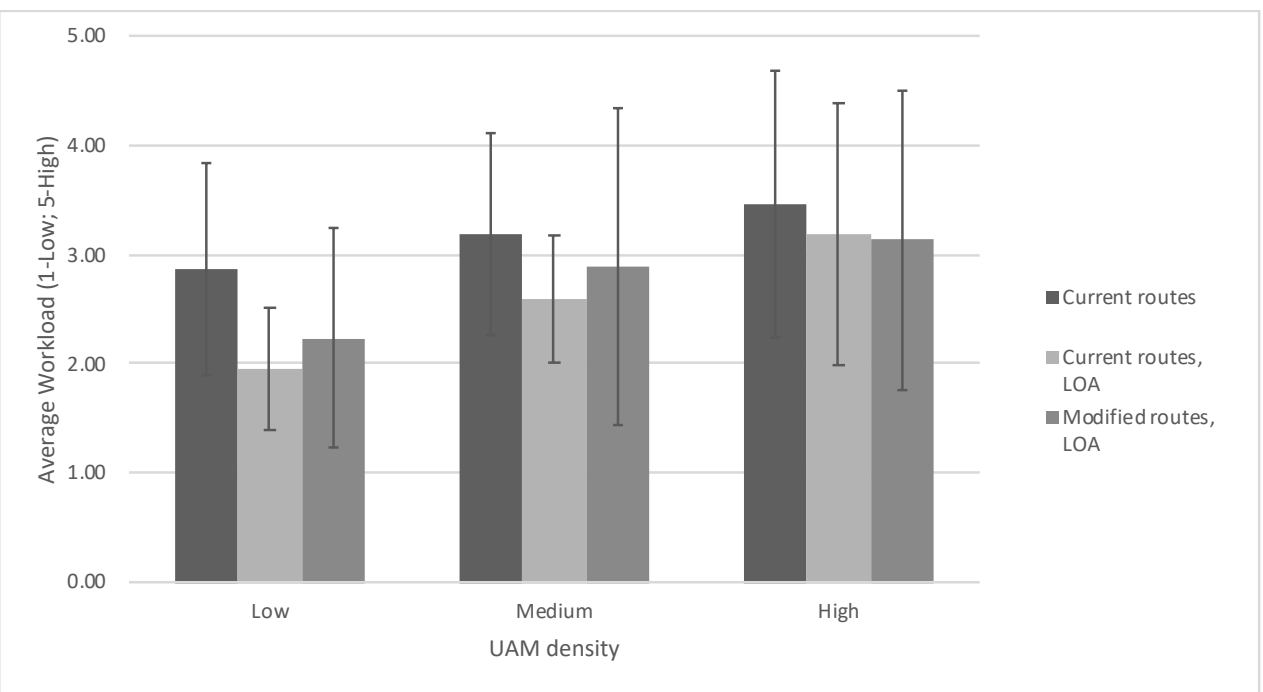

Fig. 1 Average workload across low, medium and high UAM densities and route condition for DFW Local East controller position

Figure 1 presents a comparison of workload data averaged across 40 minute runs, grouped by UAM density. As expected, it can be discerned from descriptive statistics (table 1, Figure 1) that average workload ratings increased with UAM density, suggesting that taskload affected workload as expected: $\mathrm{C} 1$ (current-day routes, no LOA, low UAM density) $\mathrm{M}=2.87, \mathrm{SD}=0.98$; $\mathrm{C} 2$ (medium $\mathrm{UAM}$ density) $\mathrm{M}=3.19, \mathrm{SD}=0.92$; $\mathrm{C} 3$ (high UAM density) $\mathrm{M}=3.45$, $\mathrm{SD}=1.22$ ). Variances are also relatively small, suggesting cohesiveness between participants' responses. The data trend of increasing average workload ratings in association with increasing UAM traffic densities is also seen in the $\mathrm{CL}$ conditions -current routes with LOA (low $\mathrm{UAM}$ density $\mathrm{M}=1.95, \mathrm{SD}=0.56$; medium UAM density $\mathrm{M}=2.60$, $\mathrm{SD}=0.58$; high $\mathrm{UAM}$ density $\mathrm{M}=3.19, \mathrm{SD}=1.20$ ). The association is again repeated in the $\mathrm{M}$ condition - Modified routes with LOA (low UAM density $\mathrm{M}=2.24, \mathrm{SD}=01.01$; medium $\mathrm{UAM}$ density $\mathrm{M}=2.89, \mathrm{SD}=01.44$; high UAM density $\mathrm{M}=3.13, \mathrm{SD}=1.37)$. It is interesting to note that the difference in workload ratings in the modified routes with LOA condition appears to be less between low, medium and high UAM traffic densities compared to the other conditions.

Inferential statistics were conducted to explore significant differences between conditions. Data were normally distributed and so parametric analysis was utilised. A repeated measures ANOVA was applied to investigate the differences in average workload ratings in association with UAM traffic density, across three routes/communication conditions (current day routes without LOA, current day routes with LOA, modified routes with LOA), to explore differences within-conditions. For the current day routes condition, Mauchly's test indicated that the assumption of sphericity had not been violated $\left(\mathrm{X}^{2}(2)=2.82, \mathrm{p}>0.05\right)$. A significant main effect of UAM traffic density was found on self-reported workload $(\mathrm{F}(2,10)=4.65, \mathrm{p}<0.05)$. Pairwise comparisons did not reveal significant differences between UAM traffic densities. However, average workload difference between low and high traffic densities approached significance $(\mathrm{p}=0.66)$. In the current day routes with LOA condition, Mauchly's test indicated that the assumption of sphericity had not been violated $\left(\mathrm{X}^{2}(2)=3.10, \mathrm{p}>0.05\right.$. A significant main effect of UAM traffic density on self-reported workload was identified $(\mathrm{F}(2,10)=9.31, \mathrm{p}<0.01)$. Paired T-Tests expanded on this finding. A Bonferroni correction was applied and so all effects are reported at a 0.02 level of significance. Pairwise comparisons revealed that workload was significantly lower in UAM low traffic density compared to medium density traffic $(\mathrm{p}=0.01)$ and high density traffic $(\mathrm{p}<0.05)$. No significant differences in average workload were found between UAM medium density and high density traffic $(\mathrm{p}=0.1)$.

For the modified routes with LOA condition, Mauchly's test indicated that the assumption of sphericity had not been violated $\left(\mathrm{X}^{2}(2)=0.29, \mathrm{p}>0.05\right.$. No significant main effect of UAM traffic density on self-reported workload was identified $\mathrm{F}(2,10)=2.36, \mathrm{p}>0.05$, possibly indicating that average workload may be more resilience to traffic increases with the use of modified routes and a LOA. 


\section{Main effect of condition:}

The following sections considers the same average workload data presented in Table 1, but focuses on the main effect of condition across each traffic density scenario (as opposed to traffic density). Regardless of conditions, traffic density within scenarios was kept constant. For example, the same UAM traffic count and density was controlled in scenario 1, low density, for C, CL and M conditions. Because objective traffic count was constant, differences in average workload ratings between conditions could not be accounted for by objective taskload, but instead, the condition itself.

When considering the low UAM density condition, there appear to be differences between $C$ (current day route, no LOA) CL (current day routes, LOA) and M (modified routes, LOA) conditions . Average workload ratings are highest in the $\mathrm{C}$ condition in $(\mathrm{M}=2.87, \mathrm{SD}=0.98)$, followed by condition $\mathrm{M}(\mathrm{M}=2.24 ; \mathrm{SD}=1.01)$, with condition $\mathrm{CL}$ recording the lowest average workload $(\mathrm{M}=1.95, \mathrm{SD}=0.56)$, potentially indicating that $\mathrm{LOA}$ has a dominant effect on reducing average experienced workload. The differences were examined for significance. KolmogorovSimonov normality checks revealed that condition C, scenario 1(low density traffics) violated the assumption of normality, and so non parametric tests were utilised with this condition only. No significant differences were found between average workload ratings in C, CL, and M conditions in the low UAM traffic scenario. $\left(X^{2}(2)=9.33\right.$, $\mathrm{p}>0.05)$.

When considering the medium UAM traffic scenario, descriptive statistics revealed the same data trend, that average workload was highest in the $\mathrm{C}$ condition $(\mathrm{M}=3.19, \mathrm{SD}=0.92)$. Average workload for the $\mathrm{M}$ condition was second highest $(\mathrm{M}=2.89 ; \mathrm{SD}=1.44)$, with average workload ratings for the $\mathrm{CL}$ condition the lowest $(\mathrm{M}=2.60$; $\mathrm{SD}=0.58$ ). The relatively large standard deviation in the modified routes condition suggests wide variability in participant responses. A repeated measures analysis of variance (ANOVA) was conducted for the medium UAM traffic scenario. Mauchly's test indicated that the assumption of sphericity had been violated $\left(X^{2}(2)=6.24, \mathrm{p}<0.05\right)$; therefore degrees of freedom were corrected using Greenhouse-Geisser estimates of sphericity $(\mathrm{E}=0.59)$. Results showed that there was a no significant main effect of condition on self-reported workload in the medium density UAM scenario $F(1.12,5.59)=1.31, \mathrm{p}>0.05)$.

Finally, descriptive statistics were reviewed for the high UAM density scenario. Descriptive statistics show that again, average workload was highest in the $\mathrm{C}$ condition (current routes with no $\mathrm{LOA})(\mathrm{M}=3.45, \mathrm{SD}=1.22$ ); average workload for the $\mathrm{CL}$ condition were second highest $(\mathrm{M}=3.19 ; \mathrm{SD}=1.20)$, and average workload for the $\mathrm{M}$ condition was lowest $(\mathrm{M}=3.13 ; \mathrm{SD}=1.37)$. The relatively large standard deviation in the modified routes condition does suggest wide variability in participant responses. A repeated measures analysis of variance (ANOVA) was conducted for the high UAM scenario. The results show that there was no significant main effect of condition on self-reported workload in the high density UAM scenario $F(2,10)=0.79, \mathrm{p}>0.05)$.

\section{Workload across conditions separated by controller position}

Although data trends were identified in average reported workload, the previous analysis used average workload across all controller positions. It was therefore important to explore average workload within each condition, to determine if any differences existed between condition. Average workload ratings were separated by controller positions and examined in relation to UAM traffic densities, across route conditions. Results will be reviewed in accordance with descriptive statistics only due to low participant numbers $(\mathrm{n}=2)$.

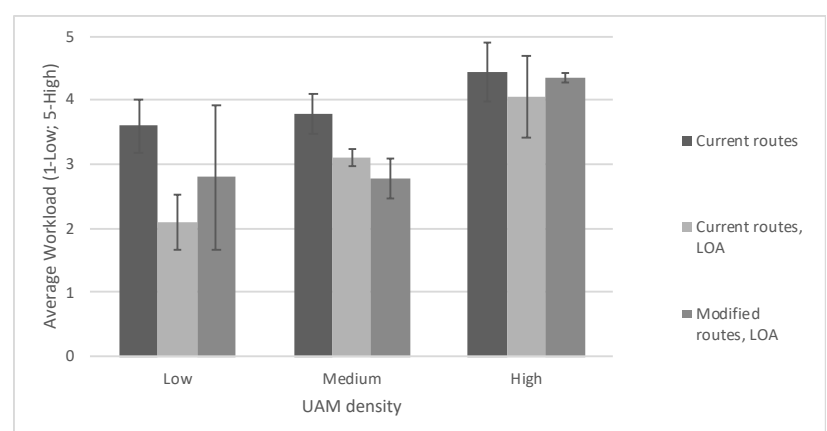

Fig. 9 Average workload across low, medium and high UAM densities and route condition for DFW Local East controller position

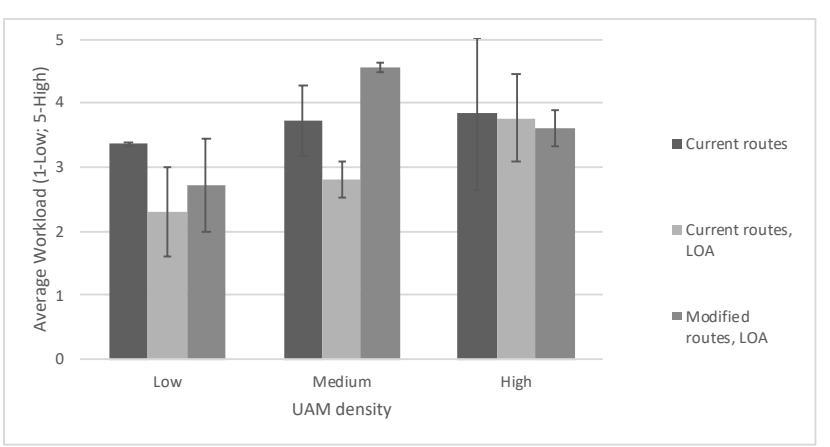

Fig. 10 Average workload across low, medium and high UAM densities and route condition for Dallas Love helo controller position 


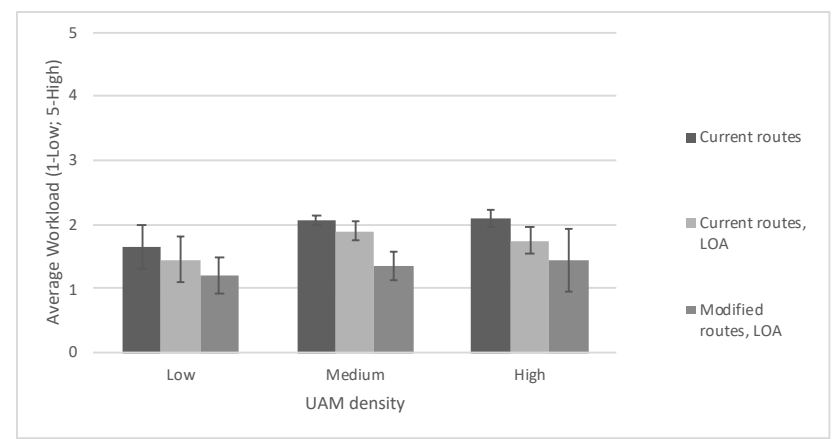

Fig. 11 Average workload across low, medium and high UAM densities and route condition for Addison tower controller position

Figure 5 presents average workload ratings from controllers working the Dallas $\mathrm{ft}$ Worth Local East Tower position. Descriptive statistics show a trend that workload ratings increase with UAM traffic density in all three conditions. A general trend can be observed where average workload increases with traffic density. Average workload was reported to be similar for both the low and medium density scenarios in the $\mathrm{M}$ conditions, even though the number of UAM traffic count increased.

Average Workload ratings by controllers working the Dallas love helo position also appear to be associated with UAM density (Figure 6). In conditions $\mathrm{C}$ and CL, workload ratings increase as UAM traffic increases. In the C condition, workload ratings appear similar between UAM low, medium and high traffic, although a small increase in workload rating with traffic is observable. In condition $\mathrm{M}$, workload ratings go against the data trend, and appear to be higher on average in the medium traffic density scenario than the high scenario, although the workload for the high density traffic remains similar for all three route conditions. Workload ratings for Dallas love helo position appear similar to DFW tower position, although on average, workload is slightly lower in the high density scenario compared to DFW.

Figure 7 presents average workload as rated by controllers for Addison tower position. A similar data trend is observed of increasing average workload with increasing UAMs. Workload overall for Addison tower position is lower compared to the other two conditions, in some cases, even by as much as half. This is due to Addison tower not controlling as much UAM traffic as the other tower positions due to the positioning of the routes.

\section{Main effect of condition}

In Dallas ft worth tower, for all density scenarios, and all controlled positions, average workload ratings were highest in the current routes condition. Minimal difference between workload rating sin the high density conditions suggest that the task demand was dominant at influencing perceived workload regardless of condition.

A similar pattern can be seen with dallas love helo tower, with rated workload highest for current routes in both low and high density scenarios. Unexpectedly, the modified routes condition resulted in the highest averge workload in medium density traffic. Although Addison tower position shows, on average, lower rated workload compared to the other control positions, there is still a clear trend in the data that suggests current routes conditions are associated with highest rated workload, suggesting a positive of both the LOA and modified routes on subjective workload.

\section{B. Throughput - UAM vehicle count}

Total throughput of UAM vehicles was recorded as indicator of efficiency-related performance associated with each condition. The total number of UAMs for each density level (low, medium, high) remained constant across all conditions. Therefore, variances in the number of UAMs controlled between conditions could not caused by variances in traffic level. An initial review of the descriptive statistics for number of UAM vehicles controlled by condition was conducted. Any participant position could deny flights into Class B airspace. If the downstream sector does not accept the handoff for a UAM flight due to their workload, it was also removed.

Verma et al. (ref) present a detailed analysis of throughput over time, but did not extend the analysis to consider total traffic count of UAM vehicles by controller position, and also did not compare these findings with selfreported workload collected throughout the simulation session. Therefore, the following results present an extension of findings building from REF.

Total traffic count averaged across controller position 
Figure xx presents the summed total of UAM traffic accepted and controlled by all control positions, for each condition, enabling general trends to be identified. UAM count was summed for each controller across the 40minute simulation period.

\begin{tabular}{|c|c|c|}
\hline & $\begin{array}{l}\text { Average } \\
\text { UAM v } \\
\text { controll }\end{array}$ & $\begin{array}{l}\text { tal } \\
\text { cles }\end{array}$ \\
\hline & $\mathrm{M}$ & $\mathrm{SD}$ \\
\hline $\begin{array}{l}\text { Current routes } \\
\text { (Baseline) UAM } \\
\text { low density }\end{array}$ & & 3.45 \\
\hline Current routes & 51.57 & 13.45 \\
\hline $\begin{array}{l}\text { (Baseline) UAM } \\
\text { medium density }\end{array}$ & 55.00 & 38.05 \\
\hline $\begin{array}{l}\text { Current routes } \\
\text { (Baseline) UAM }\end{array}$ & & \\
\hline high density & 63.33 & 38.55 \\
\hline $\begin{array}{l}\text { Current routes, } \\
\text { LOA, UAM low }\end{array}$ & & \\
\hline $\begin{array}{l}\text { density } \\
\text { Current routes. }\end{array}$ & 47.17 & 29.89 \\
\hline $\begin{array}{l}\text { LOA, UAM } \\
\text { medium density }\end{array}$ & 70.00 & 41.36 \\
\hline $\begin{array}{l}\text { Current routes, } \\
\text { LOA. UAM high }\end{array}$ & & \\
\hline density & 92.00 & 60.00 \\
\hline $\begin{array}{l}\text { Modified routes, } \\
\text { LOA, UAM low } \\
\text { density }\end{array}$ & 50.50 & 5.60 \\
\hline $\begin{array}{l}\text { Modified routes, } \\
\text { LOA, UAM }\end{array}$ & & \\
\hline medium density & 71.17 & 47.50 \\
\hline $\begin{array}{l}\text { Modified routes, } \\
\text { LOA, UAM High } \\
\text { density }\end{array}$ & 96.00 & 68.87 \\
\hline
\end{tabular}

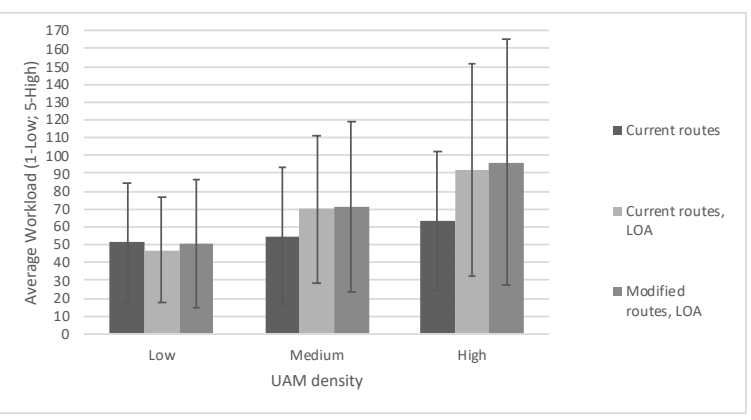

1. Main effect of scenario

From a review of figure $\mathrm{xxx}$ it appears that, as expected, mean total of UAM vehicles controlled did increase across density scenarios for all conditions. (Table XX, figure XX). A repeated measures ANOVA was conducted to compare differences between conditions. No significant differences were identified between total number of UAM vehicles controlled between low, medium and high traffic scenarios for condition C (current routes without LOA) ( $F(2,10)=2.12, \mathrm{p}=0.17)$. A significant difference was found for total UAM vehicles controlled between low, medium high density scenarios for the CL condition (current routes with LOA) $(F(1.02,5.08)=12.30, \mathrm{p}<0.05$.) 
Pairwise comparisons were conducted. A Bonferroni correction was applied and so all effects are reported at a 0.02 level of significance. Pairwise comparisons revealed that significantly less UAM vehicles were controlled in the low density condition than the medium density condition $(p<0.01)$ and high density condition $(p=0.02)$. the difference in controlled UAM traffic was not significantly greater in the high density condition compared to the low density condition $(\mathrm{p}=0.04)$. Considering condition $\mathrm{M}$ (modified routes with LOA), a significant difference was identified for total UAM vehicles controlled between low, medium high density scenarios $(F(1.34,5.17)=8.36$, $\mathrm{p}<0.05$ ). Pairwise comparisons were conducted. A Bonferroni correction was applied and so all effects are reported at a 0.02 level of significance. Pairwise comparisons revealed that significantly less UAM vehicles were controlled in the low density condition than the medium density condition $(\mathrm{p}=0.01)$ and approached significance between the high density traffic $(p=0.03)$. No significant differences between workload ratings were found between UAM medium density and high density traffic $(\mathrm{p}=0.07)$.,

\section{Main effect of condition}

A finding of note is that although UAM traffic remained constant within density scenarios, the total count of UAM traffic controlled throughout each condition, within traffic density scenario, was different. It is evident from table $\mathrm{xx}$ figure $\mathrm{xx}$ that similar levels of UAM traffic were controlled in the low density scenario across condition $\mathrm{C}$ (current route) $(\mathrm{M}=51.57, \mathrm{SD}=33.45)$, condition $\mathrm{CL}$, (current route with $\mathrm{LOA})(\mathrm{M}=47.17, \mathrm{SD}=29.89)$ and condition $\mathrm{M}$ (modified routes with LOA) $(\mathrm{M}=50.50, \mathrm{SD}=35.60)$. This suggests that controllers managed to control all UAM flights that were available in the low density scenario. In the medium density scenario, fewer UAM vehicles appear to have been controlled in the $\mathrm{C}$ condition $(\mathrm{M}=55.00, \mathrm{SD}=38.05)$ compared to the $\mathrm{CL}(\mathrm{M}=70.00 ; \mathrm{SD}=41.36)$ and $\mathrm{M}$ condition $(\mathrm{M}=71.17 ; \mathrm{SD}=47.40)$. A relatively large difference is seen in the high density scenario, in which fewer UAM vehicles are managed on average in the $C$ condition $(m=63.33, S D=38.55)$ than the $C L](M=92, S D=60)$ or the $\mathrm{M}$ condition $(\mathrm{M}=96.00, \mathrm{SD}=68.87)$. Results indicate that controllers managed to control more traffic in in $\mathrm{CL}$ and $\mathrm{M}$ conditions compared to the current day routes. A repeated measures ANOVA was conducted to test differences of significance for each density scenario. No significant differences in total UAM vehicles controlled were found between $\mathrm{C}, \mathrm{CL}$ and $\mathrm{M}$ conditions in the low density scenario. $(F(2,10)=0.50, \mathrm{p}>0.05)$. In addition, the differences between controlled traffic in the medium density scenario were also not significant $(F(2,10)=2.78$, $\mathrm{p}>0.05)$. Differences between traffic managed were significant in the high density scenario $(F(2,10)=5.05, \mathrm{p}<0.05)$. Pairwise comparisons were conducted. A Bonferroni correction was applied and so all effects are reported at a 0.02 level of significance. Pairwise comparisons revealed that significantly less UAM vehicles were controlled in the low density scenario than the medium density scenario $(\mathrm{p}<0.001)$ and high density scenario $(\mathrm{p}<0.01)$. More vehicles were also controlled in the modified routes condition compared to the current routes with LOA $(p<0.005)$

\section{By controller position}

It is evident from the wide standard deviations (Range $=29.89-68.87$ ) that there was large variation in the UAM traffic counts that were controlled by each controller position. In order to explore UAM vehicle count controlled further, the data were separated by controller position. Table XX presents the average sum of all UAM vehicles accepted to be controlled in each conditions, separated by controller position.

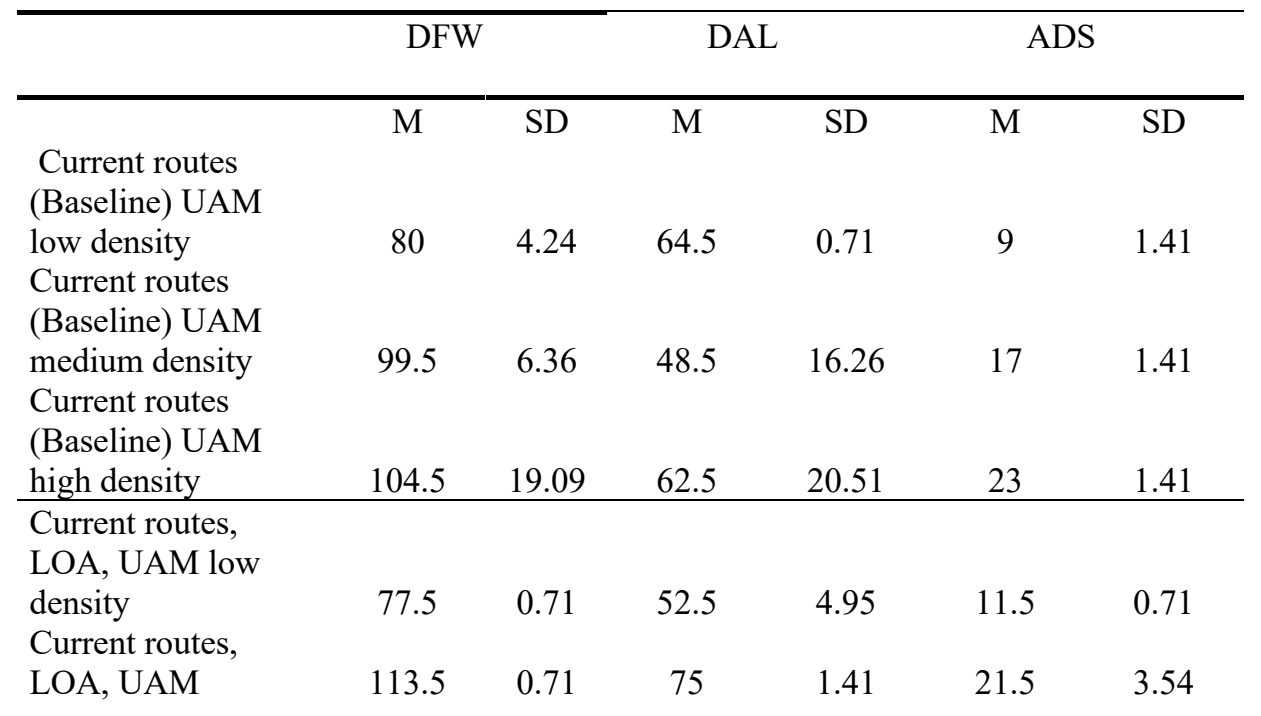


medium density

Current routes,

LOA, UAM high

density

LOA, UAM low

density

$158.5 \quad 9.19$

92.5

6.36

25

7.07

Modified routes,

$74 \quad 11.31$

72

11.31

5.5

0.71

LOA, UAM

medium density

107

11.31

95.5

12.02

11

4.24

Modified routes,

LOA, UAM High

density

$\begin{array}{ll}161.5 & 9.19\end{array}$

11

15

7.07

11.

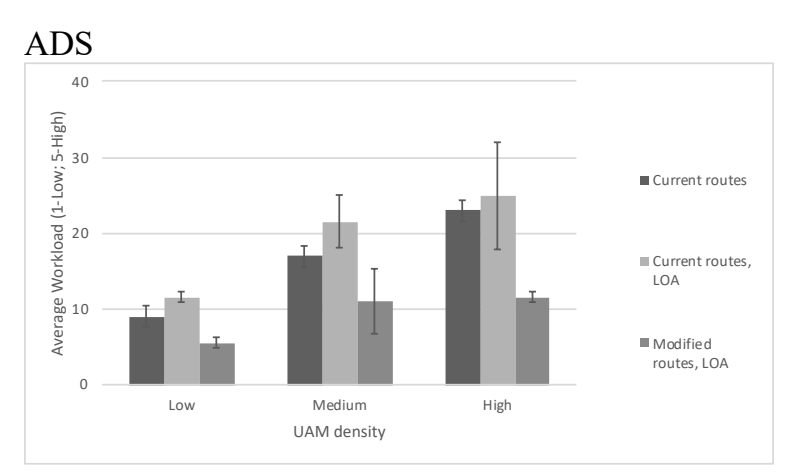

DAL
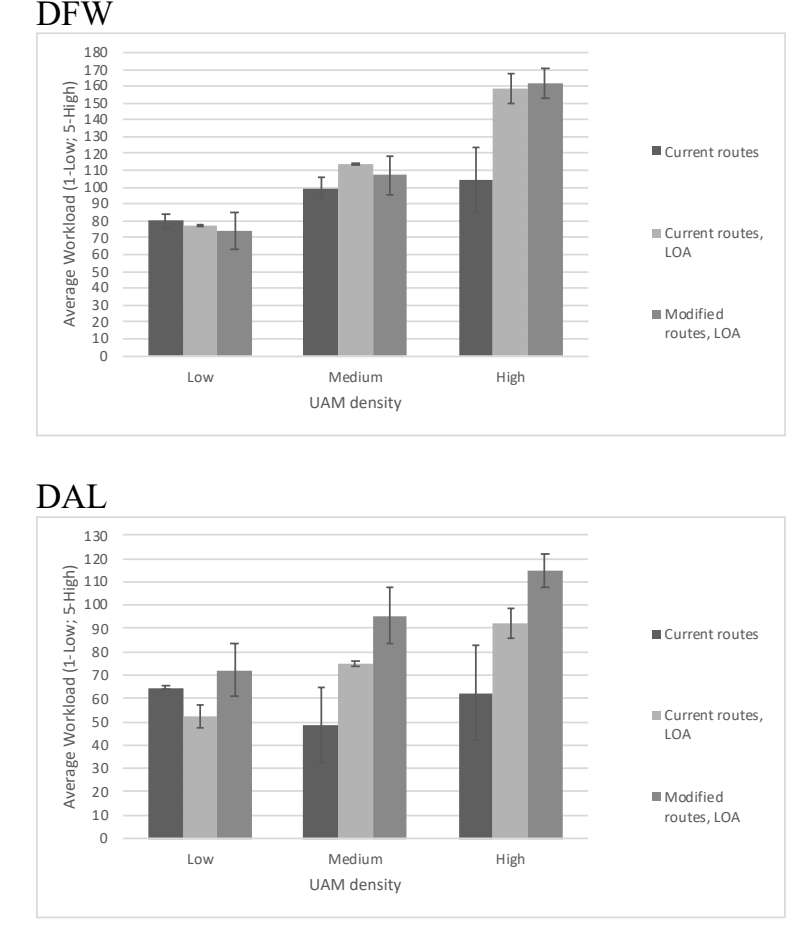

A review of table xx and figures xx reveals that the pattern of controlled UAM counts is similar for DFW and DAL. Traffic counts are similar across conditions in the low density scenario, with more obvious differences in traffic counts for medium scenario and high scenarios. Inferential test of significance were not possible due to the small $n$ of each controller group.

\section{A note on the Relationship between workload and UAM controlled}

As the level of traffic in the scenario was assumed to be one of the main influences on workload, the relationship between perceived workload and UAM control was investigated for each scenario across condition. Due to the large number of results, not all data is represented in this paper. The relationships between workload and average UAM vehicle count was investigated using Spearman's correlations (due to a violation of independence). As a way of succinctly presenting the data, table xx shows the Spearman's correlation coefficient and associated significance level. As in the rest of the results, controller positions were first collapsed in order to examine the overall relationship.

\begin{tabular}{cc}
\hline Spearman’ & Significanc \\
s & e \\
coefficient &
\end{tabular}




\begin{tabular}{|c|c|c|}
\hline $\begin{array}{l}\text { Current routes } \\
\text { (Baseline) UAM } \\
\text { low density } \\
\text { Current routes } \\
\text { (Baseline) UAM } \\
\text { medium density } \\
\text { Current routes } \\
\text { (Baseline) UAM } \\
\text { high density } \\
\end{array}$ & 0.63 & $\mathrm{p}<0.001$ \\
\hline $\begin{array}{l}\text { Current routes, } \\
\text { LOA, UAM low } \\
\text { density } \\
\text { Current routes, } \\
\text { LOA, UAM } \\
\text { medium density } \\
\text { Current routes, } \\
\text { LOA, UAM high } \\
\text { density } \\
\end{array}$ & 0.79 & $\mathrm{p}<0.05$ \\
\hline $\begin{array}{l}\text { Modified routes, } \\
\text { LOA, UAM low } \\
\text { density } \\
\text { Modified routes, } \\
\text { LOA, UAM } \\
\text { medium density } \\
\text { Modified routes, } \\
\text { LOA, UAM High } \\
\text { density }\end{array}$ & 0.68 & $\mathrm{p}<0.001$ \\
\hline
\end{tabular}

As presented in table $\mathrm{xx}$, all relationships were significant. This suggests that there is a close covariance between workload and number of UAM vehicles controlled. Interestingly, in the CL condition, there is a moderately singulative negative correlation between workload and traffic count, where workload increased as less traffic is controlled. This may indicate reduced efficiency in this condition under high destiny traffic. In order to assess the relationship between workload and traffic for separate control positions, the relationship was also examined using spearman's correlations. No relationships were found to be significant at the 0.05 level.

\section{Workload and performance efficiency: percentage of UAM vehicles controlled}

Another method of analyzing efficiency permeance was to consider the percentage of UAM aircraft accepted and controlled was calculated by condition for each scenario. The vehicle was counted throughout the session and was counted if controllers accepted control of the UAM vehicles. In order to understand the association between workload and performance metric, these were considered together. Control positions were first considered together.

Figures xxx present the average workload and percentage of accepted UAM vehicles for every condition across density scenario.

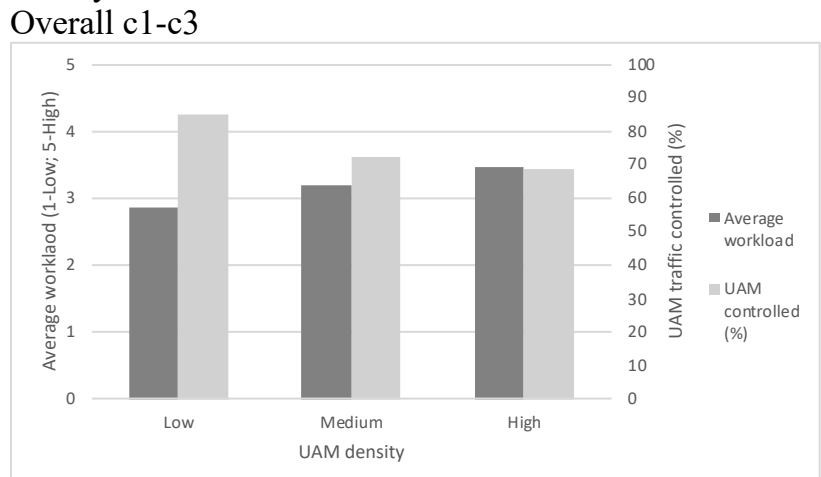

Overall cl1-3

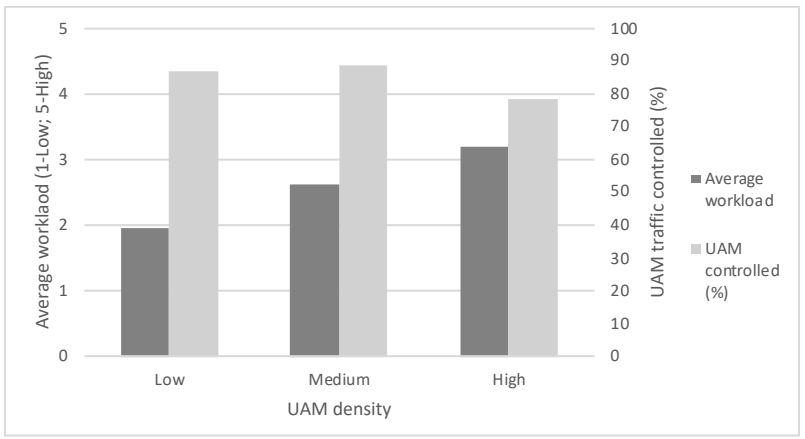

Overall m1-3 


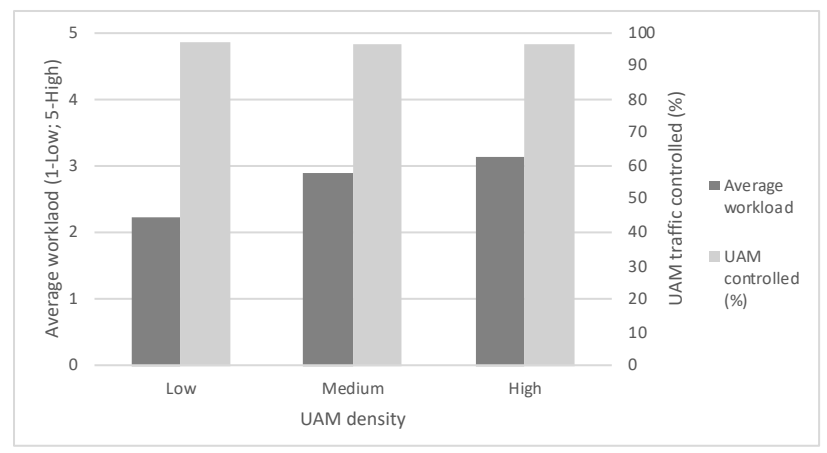

In condition 1, with current routes, workload is rated around the mean point of the scale $(\mathrm{M}=2.87, \mathrm{SD}=0.98)$ with a high percentage of controlled UAM $(\mathrm{M}=84.75 \%)$. In the medium density scenario, workload increases $(\mathrm{M}=3.19$, $\mathrm{SD}=0.92$ ), although UAM \% controlled dropped to 72.18. Finally, in the high density scenario, workload increases further to an average of 3.45, whilst percentage of UAMs managed dropped to 68.45. together, these results suggest that as traffic density increases with these current routes, workload rises and the amount of traffic controlled drops, potentially due to controllers not accepting more aircraft into the airspace.

This is in comparison to the CL condition. Percentage of UAM controlled aircraft is also higher in each density scenario compared to condition 1 (low=86.74\%, medium $88.79 \%$, high $78.15 \%$ ). There is also a more positive association that even as workload rises in accordance with the density conditions, UAM traffic controlled performance is not negatively affected. In the highest density scenario, performance efficiency does drop compared to the low and medium conditions. This may suggest that with the current routes with LOA, more traffic can be managed, and workload is reduced, in the low and medium conditions, but in the high condition, there is still too much traffic to work with and so some refusals or non-management occurs.

Finally, when considering the modified routes. Workload is rated still lower (low= medium=high=) but all $\%$ are also high, at $96 \%$. Efficiency does not change across densities, even with the workload rating increasing. this finding suggests that the modified routes are effective at lowering workload and improving efficiency. In addition, this is seen to also be most effective in the high density coition. In the lower density conditions, current route with reduced verbal communication is sufficient, but only the modification of routes plus the loa is sufficient for reducing workload.

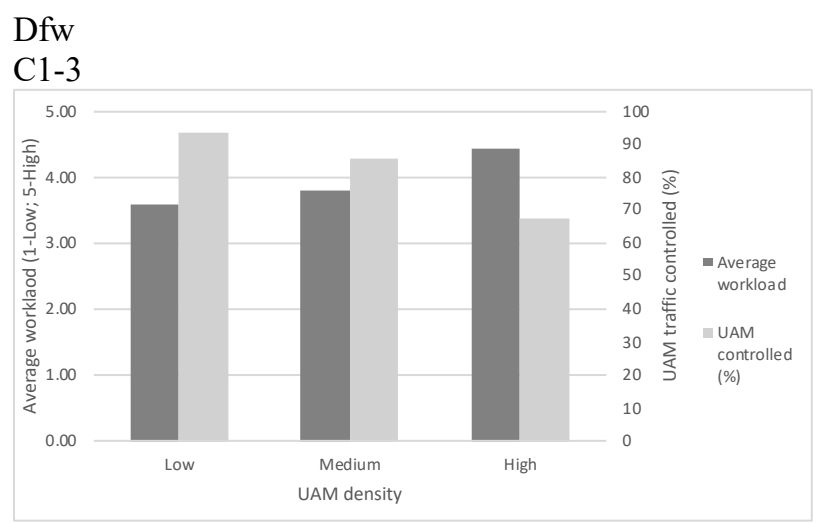

Dfw cl1-3

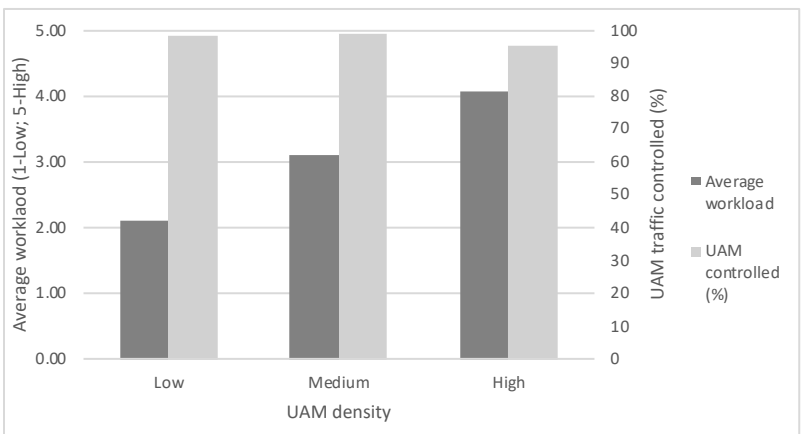

Dfw m1-3

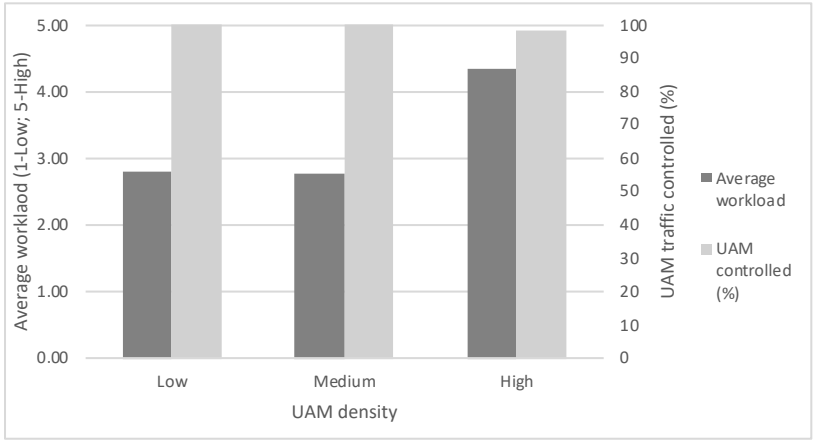


Dal

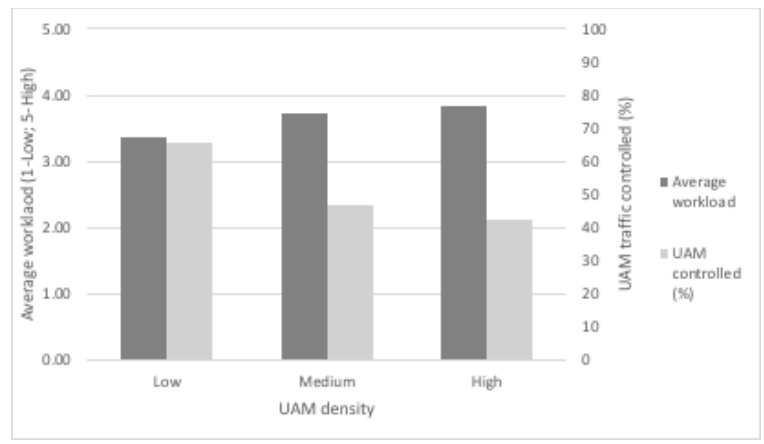

\section{Dal cl1}

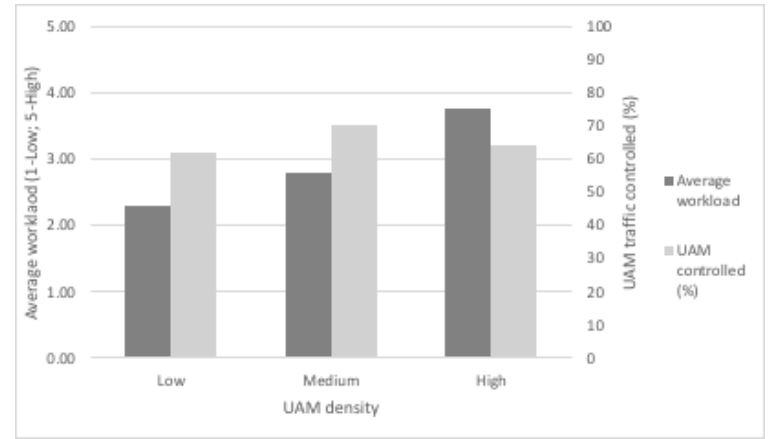

M1

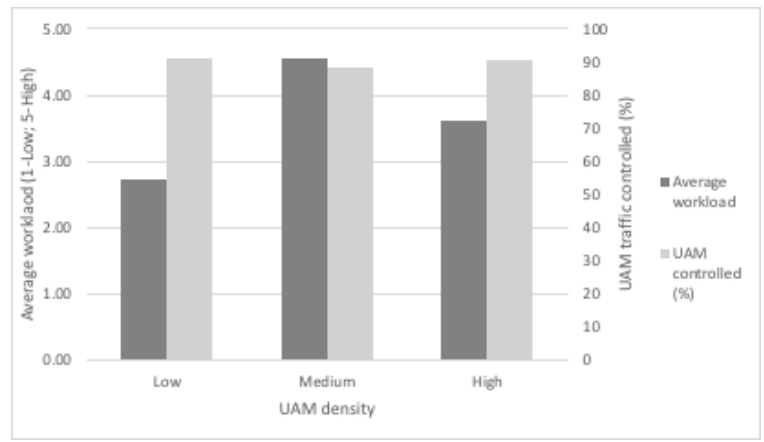

Ads c1

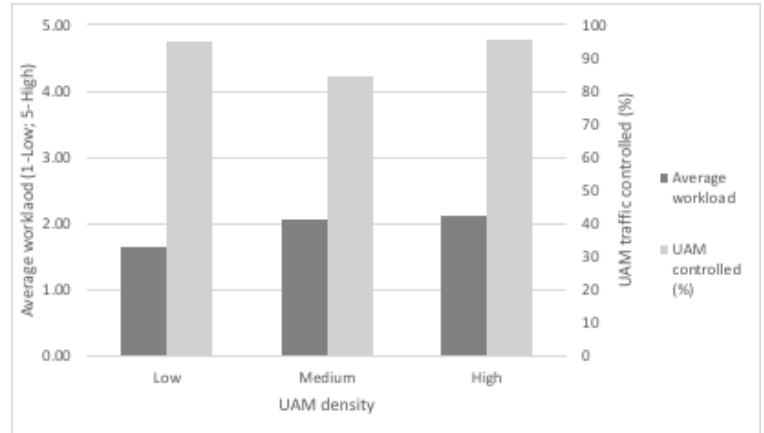

C11

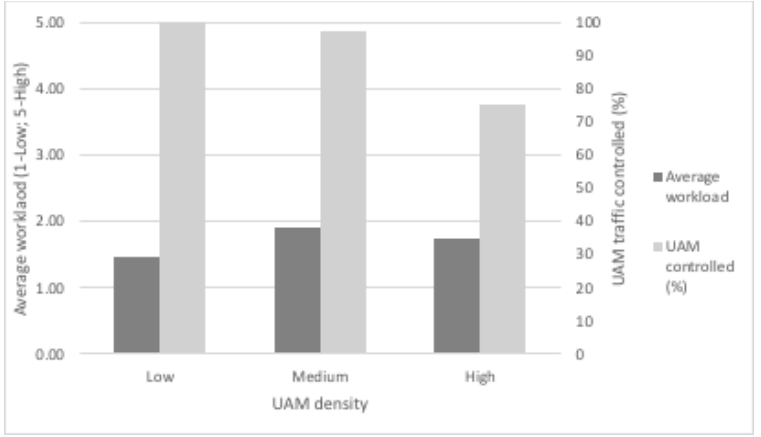

M1

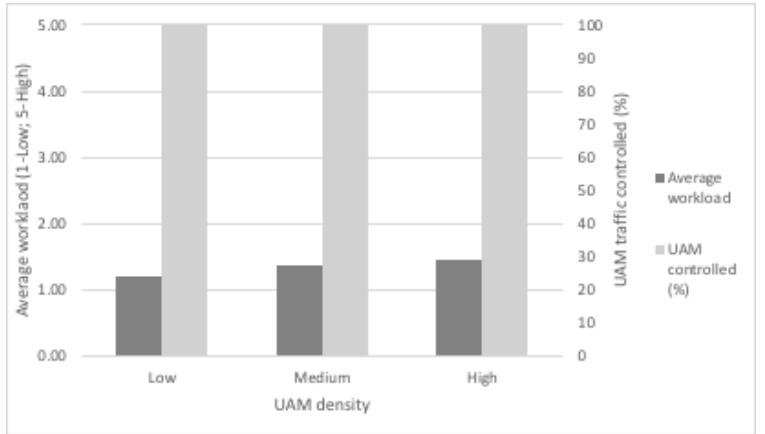




\section{Discussion}

A human in the loop air traffic control simulation was used to investigate the effect of UAM traffic density and changes in current airspace routes and communication procedures on subjective controller workload and efficiencyrelated task performance. The study aligned with a potential near-term concept for integration of UAM traffic into the current airspace, and assumed active human-centric control and a centralized system of operations, similar to the current ATM environment for commercial aircraft. Current procedures and regulations surrounding class B,C and D airspace were incorporated into the simulation. Controllers were responsible for all tasks as per the current day. The aim of the study was to investigate the human factors issues in a positive and human-centric control concept, and potential areas of concern, as well as whether it was feasible near-term step in the interaction of UAM traffic into the current airspace. secondly, the study aimed to explore if modifications to the currently used routes and communication procedures for class b airspace would positively influence human factors issues such as workload and efficiency performance metrics.

\section{A. Workload, density and route/communication conditions}

An analysis of average self-reported workload, collapsed across controller positions, revealed a pattern that workload varied with traffic density, across all conditions. This suggests that UAM demand created variability in self-reported workload, and demonstrates, as expected, that increasing UAM traffic is positively associated with subjective workload. In condition $\mathrm{C}$, workload differences in the low and high density traffic scenarios approached significance. Workload was also rated significantly higher in the high density compared to low density scenarios. An interesting findings was that differences in workload ratings did not reach significance in the modified routes condition. Variances were larger in this condition possibly weakening the inferential analysis, as the range was similar both other conditions.

When separated between controller positions, the same pattern was seen repeatedly. It was evident from a review of descriptive statistics across condition that of the control positions that Addison tower position overall perceived the lowest workload. Due to the position of Addison tower, fewer routes, and shorter routes passed through this airspace. For real operations, it is important to note that depending on the positioning of the UAM traffic routes, workload distribution between controller positions may not be assumed to be equal. Instead, route placement should be considered carefully due to workload implications. In addition, Addison was also in Class C airspace, resulting in fewer regulations. This also may have reduced experienced workload. Controllers in the Dallas $\mathrm{ft}$ Worth tower position on average rated highest workload, suggesting that in future studies, particular attention should be focused on this position. Workload has been repeatedly found to significantly correlate with performance influencing factors such as stress, situation awareness decline and increased fatigue (REF). Therefore, an important area of continued research is workload reduction if UAMs are to be actively controlled. The data were then analyzed in terms of differences between condition.

The analysis considered workload rating for each condition (C, CL, M) in association with each traffic density scenarios. Within scenario, traffic levels remained constant for each condition. Overall, when collapsed across controller position, a review of the descriptive statistics in the low density scenarios showed that there were differences in average perceived workload even though objective traffic counts remained constant. Workload was rated higher in the current route condition than the other conditions. This suggests that even though traffic remained constant, controllers had to work harder to control traffic than in the CL or M conditions. On average, the modified routes were rated higher in workload than the CL condition. This difference was marginal, however. This may be have reflected that the modified routes, although optimized for UAM vehicles, may have created additional workload in some way. However, the large variances across the modified routes condition in all scenarios suggest that controllers experienced the Modified routes condition differently, which influenced workload. No differences between workload ratings between conditions were significant, however. Although all workloads were rated higher in the high density condition compared to low density, average workload were still rated higher in the current route condition than other conditions, although differences were not significant. Interestingly, in the high UAM density scenario, the pattern changes slightly so that the current route condition is the highest workload and modified routes is lowest. The difference between this and the current routes with LOA is marginal, but it may suggest that under high conditions, modified routes may work more effectively to reduce workload than under lower task demand scenarios. Differences were not significant.

It was acknowledged that there were large variances in the data, especially in the high workload condition. Therefore, data were separated between controller positions. For Addison position, workload was consistently rated as low. The same data trend was seen consistently, although the $\mathrm{C}$ condition was always rated to have higher 
workload than either the CL or M condition. This suggests that even in periods of lower workload, the LOA and modification of routes still have a positive impact on workload. However, the range of means between condition per scenario were relatively small for all conditions, suggesting that the positive impact was not as marked as in other conditions with higher demand.

A data trend was identified that suggested current routes and procedures, without a LOA or other form of agreement, are not suitable for UAM integration in controlled airspace for scaled operations. Specifically, for the DFW position, the M condition was rated higher for workload than the CL condition. This may have been due to lac of familiarity with routes. In summary, findings suggest that for all positions and traffic levels, routes with current procedures resulted in the highest reported subjective workload for controllers. This suggests that the traditional procedures and regulations may limit operations for any near-term development with human controllers due to workload.

\section{B. Throughput by condition}

Descriptive statistics of the total number of UAMs accepted for control by participants revealed that as expected, the number of UAMs controlled increased with traffic demand. Considering the average sum of UAM vehicles controlled within scenario, there are again differences in the total of UAMs controlled, even with UAM traffic held constant. This is because controllers could elect to refuse entry to the eVTOLs if they believed permitting entry into controlled airspace would decrease performance and overload them.

Within the low condition, a similar number of eVTOLs on average were controlled, supported by inferential statistics which showed no significant differences between the number controlled was found.

In the medium density condition, overall, significantly less eVTOLs were accepted for control on average in the current route condition compared to the CL and M conditions. Combined with self-reported workload data, this confirms that less aircraft were potentially accepted for control due to the higher experience workload. However, this also suggests that the current route condition, at medium density, create such as demand that controllers were rejecting UAMs form entering controlled airspace. Even at medium density, the demand was judged to be too great to safely control. Comparisons with the other conditions show that highly similar number eVTOLs were controlled in CLA and modified conditions, and inferential statistics confirmed that the difference between conditions was not significant. This sis interesting to compared with rated workload, as even though more aircraft were handled compared to current routes, workload was reported to be less. This suggests a positive effect of the communication $\mathrm{d}$ route changes. Although the direction of the relationship cannot be determined with confidence/causality, it may be that the changes allowed a reduction of workload and therefore increases in efficiency.

In the high-density scenario, the data trend is even more apparent. The number of eVTOL controlled in the current condition remained similar across all scenarios, suggesting a maximum limit for the current routes was reached, and aircraft were rejected no matter that the task demand increased. Significantly less eVTOLs were managed in the current route scenario compared to CL, and approached significance with modified routes. Again, there was no significance difference between modified and CL routes, suggesting that the real impact of reduced workload and increased efficiency was the reduction in verbal communication rather than the optimized routing, although this may have a positive impact for eVTOLs.

A comparison of descriptive data broken out by each controller position revealed the same data trend for all positions. For all conditions, current routes managed were lower. In DFW, the average number of eVTOL managed was similar in all densities between condition CL and M. However, interestingly, in Dallas Love, there were larger differenced, with modified routes having more eVTOL than CL. This is an interesting distinction, as it reveals that the modified routes were more beneficial to the Dallas love position compared to DFW. The collapsing and averaging across positions masked this data trend. This, however, makes sense - the routes modified too workload away from Dallas love but not from the DFW controller. Therefore, modified and CL routes were similar for DFW as reduced communications had bigger impact. However, when considering the modification of routes so that the controller has less routes to manage, less complexity, then this also has a significant effect on the number of eVTOL that can be controlled, potentially above the communications. Similarly, in Addison, the modified routes resulted in a lower number of eVTOLs were managed than the other conditions as routes were moved away from the Addison sector/area of control. Explains the large variances.

Overall, it suggests that LOAs can significant increase efficiency and number of eVTOL possible to control, supposedly due to a reduction in workload (as per previous findings section). Depending on the characteristics of route modification, this may have a larger impact than reduction through LOA, but if characteristics generating workload are not reduced, increases complexity or is two close to existing routes, then the impact will not contribute or may even increase workload and reduce effects of reduced communication. These suggest that not only positively affect workload but also increase efficiency performance. 


\section{Relationships between workload and throughput}

Although covariance between workload and throughput was assumed, it was important to establish if a correlation relationship existed and in what form. This was to serve as a check on the task demand creating variance in perceived workload covariate. When grouped together, on average, significant positive relationships were found across all scenarios for all groups, the only exception to his was a significant negative correlation between current CL condition, high density scenario, in which traffic went up but workloadwent down This may be due other effect of the LOA in high density traffic, which reduced workload while allowing an increase in traffic. It may also be a fallacy.

Tis findings suggested that task demand and workload were significantly related which served as a check on the study design that taps demand did create variance in workload. However, when exploring by controller position, no relationships were found, this could be due to less $\mathrm{n}$ and lower power, but also on visual inspection of the data, curvilinear relationship were identified in which workload traffic count would initial raise with workload, but then workload would decrease and traffic count still up due to the condition. Therefore, the correlation analysis may not be fully representing the data. To investigate further, the relationships between workload and percentage of aircraft controlled was examined, this did not rely on correlation analysis and so would not be affected by curvilinear relationships. In addition, as a performance metric it may be more information than throughput/count alone

\section{Workload, routes and task performance}

Task performance was assessed by the percentage of UAMs accepted for control. Overall performance was good, with most aircraft being controlled between $80-100 \%$. Overall, in current route conditions, \% UAM controlled aircraft reduced as density. And reported workload increased. This suggests that there was too much traffic to safely handle in the medium and high density conditions, with $84 \%$ moving to $68 \%$.

A similar data trend as apparent in the CL, although the range was much lower, between 88-78).

Interestingly, similar \% of traffic worked was found between low and medium density, and only dropped to 78 in high. It suggests that although workload was still rising, overall the range of workload was lower than in the current day condition, and so the increase in workload due to demand did not appear to influence $\%$ worked until the highest density traffic scenario.

It is interesting to note that in the modified route condition, overall, that even though workload increased over density, \% aircraft worked remained consistently high. Taken together with the finding of number of UAM traffic through number of aircraft worked, this is an interesting result. Previous, number of aircraft work seemed to depend on controller position and modified routes different if as useful as reduction in communications. So this results adds information to saddest that overall, even though efficiency may have been similar and workload similar, controllers were more likely to consistently work higher \% of traffic, suggesting that modified routes made it more likely that it is possible to work the traffic given in high density, compared to C and CL.

Overall, findings suggest that positive effect from both CL and M condition, although may be operating on two different mechanisms for workload and increasing task performance.

\section{E. Implications and future research}

It is acknowledged that these results are provisional, and need to be interpreted within context. For example, in an air traffic environment, it is easier for the controller to build a picture of the traffic by ramping up with the traffic rather than just starting a session in a high demand period [6]. However, findings do have important implications for the prediction of controller performance in an operational environment. Findings suggest that high and low demand periods can affect controller perception of covariate factors such as workload differentially depending on what has happened prior to the current situation. Thus, supervisors may need to pay close attention to the number and direction of transitions that a controller experiences per session to most effectively support controller performance.

Future research should further explore the relationship between previous task demands and the relationship on present controller experience, including the exploration of sudden, and unexpected, transitions. Better predictions are needed to identify and prevent potential performance declines and associated performance-related incidents. Such predictions may be particularly relevant for adaptive automation technologies that support operator performance.

\section{Conclusion}

The effect of task demand transitions on workload and one, efficiency related performance measure was investigated within the context of an air traffic control task. Findings indicate that although experienced workload was manageable for low density operations, medium an high density operations let to unmanageable levels of workload, 
leading to refusals to allow more vehicles into controlled airspace. By implementing a letter of agreement, verbal communications which were associated with reduced workload. Optimized routes, reducing complexity of traffic mixes, was also associated with reduced workload and increased performance efficiency, as measured by percentage throughput of controlled traffic. Although these adjustments can positively support controller performance, workload still remained high during the high density UAM traffic scenarios. It is therefore suggested that, in order for UAM operation to become scalable, human operators will need to have altered responsibilities compare to current day air traffic controllers. Future research should therefore focus on the level and type of human operator or controller involvement, interaction with automated systems, whilst maintaining safety levels in aviation.

\section{References}

${ }^{1}$ Thipphavong, D.P., Apaza, R., Barmore, B., Battiste, V., Burian, B., Dao, Q., Feary, M., Go, S., Goodrich, K.H., Homola, J. and Idris, H.R., 2018. Urban Air Mobility Airspace Integration Concepts and Considerations. In 2018 Aviation Technology, Integration, and Operations Conference (p. 3676).

${ }^{2}$ Courtin, C., Burton, M.J., Yu, A., Butler, P., Vascik, P.D. and Hansman, R.J., 2018. Feasibility Study of Short Takeoff and Landing Urban Air Mobility Vehicles using Geometric Programming. In 2018 Aviation Technology, Integration, and Operations Conference (p. 4151).

${ }^{3}$ Chan, W.N., Barmore, B., Kibler, J., Lee, P.U., O'Connor, N., Palopo, K., Thipphavong, D.P. and Zelinski, S., 2018. Overview of NASA's ATM-X Project. In 2018 Aviation Technology, Integration, and Operations Conference (p. 3363).

${ }^{4}$ Lowry, M., 2018. Towards High-Density Urban Air Mobility. In 2018 Aviation Technology, Integration, and Operations Conference (p. 3667).

${ }^{5}$ Vascik, P.D. and Hansman, R.J., 2017. Constraint Identification in On-Demand Mobility for Aviation through an Exploratory Case Study of Los Angeles. In 17th AIAA Aviation Technology, Integration, and Operations Conference (p. 3083).

${ }^{6}$ Tattersall AJ, Foord PS (1996) An experimental evaluation of instantaneous self-assessment as a measure of workload. Ergonomics 39(5):740-748

${ }^{7}$ Prevot, T., Lee, P., Callantine, T., Mercer, J., Homola, J., Smith, N. and Palmer, E., 2010. Human-in-the-loop evaluation of NextGen concepts in the Airspace Operations Laboratory. In AIAA Modeling and Simulation Technologies Conference (p. 7609). 
American Institute of Aeronautics and Astronautics 